scripts containing answers of 'No' to the first two questions and 'Yes' to the third question were then analysed in terms of the number of similarities and of differences in the comparisons made. The results, with three groups of observers, were as follows :

\begin{tabular}{|c|c|c|c|c|c|c|}
\hline No. of observer & & . & $\cdots$ & 8 & 11 & 9 \\
\hline No. of pairs of & bjects & . & .. & 3 & 5 & 4 \\
\hline No. of points of & compar & ison & $\cdots$ & 112 & 230 & 131 \\
\hline Similarities & .. & .. & . & 34 & 53 & 20 \\
\hline Dissimilarities & .. & .. & .. & 78 & 177 & 111 \\
\hline
\end{tabular}

It would appear, therefore, that when unprejudiced observers spontaneously make comparisons between pairs of objects, they tend to note many more dissimilarities than similarities.

Those who are accustomed to study scientific method from a philosophical as distinet from a scientific point of view may be inclined to criticize these results, saying that more dissimilarities than similarities are noted in a given time because the objects possess more dissimilarities. But a scientific study of a subject must be based upon observables. How, in terms of observables, are we to know the total number of similarities and dissimilarities possessed by a pair of objects? When attention was directed to many unnoted similarities, they could all be recognized as such by the observers taking part.

In biological experiments variations are invariably found with the individual. It is therefore significant that for no unprejudiced observer was the number of similarities equal to or greater than the number of dissimilarities. Of prejudiced observers, six who tried to include only similarities, gave 95 points of comparison, of which 14 were dissimilarities, in spite of the effort to include similarities only. Two other prejudiced observers, who tried to include dissimilari. ties only, gave no similarities. The mean numbers of points of comparison per person for each pair of objects were :

\begin{tabular}{|c|c|c|c|}
\hline Unprejudiced & & & .. \\
\hline Dissimilarities only & . & $\cdots$ & . \\
\hline Similarities only & 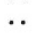 & .. & . \\
\hline
\end{tabular}

Although these results are presented here for their relevance to the scientific study of scientific method, they would appear to have some bearing also on social problems. Not only do some individuals show great talent for selecting differences in dealings with their fellow men, but also, in international affairs, nations at the present time are stressing a few points of difference instead of their many points of similarity.

I should be grateful to hear of any other experiments bearing on the perception of similarity or the scientific study of scientific method.

W. H. George.

University College,

Southampton.

May 11.

\section{Karrer's Theory of Coupling}

IN 1915, Karrer ${ }^{1}$ put forward the view that the coupling of aromatic tertiary amines with diazo. compounds to form alkylamino azo-compounds takes place through the preliminary combination of the diazo-compound with the nitrogen of the amino group. The experimental basis for this theory was the reported observation that when either di-butylaniline or di-isoamylaniline reacts with diazo. sulphanilic acid, an alkyl group is eliminated with the formation of the corresponding monoalkylamino azobenzene sulphonic acid.
It is unfortunate that Karrer did not specify more exactly which of the di-butylanilines he employed. We have repeated Karrer's experiments with both the dibutylanilines described in the literature, namely, di- $n$-butylaniline and di-isobutylaniline. Both are found to couple normally without the loss of a butyl group. This confirms an earlier observation by Reilly and Hickinbottom ${ }^{2}$ that di- $n$-butylaniline reacts normally with diazosulphanilic acid. It is also found that diazosulphanilic acid couples with diisoamylaniline without the loss of an alkyl group.

It is concluded that Karrer's observations were at fault and that there is no experimental basis for Karrer's theory of coupling. This conclusion is supported by the observation that methyl tertbutylaniline also couples with diazo-compounds without the loss of an alkyl group, although it has been shown earlier that the tert-butyl group is only loosely bound to the nitrogen ${ }^{3}$.

During the course of this work, it was also found, eontrary to Karrer's observations, that the two dibutylanilines and di-isoamylaniline react with nitrous acid in aqueous acid solution to form $p$-nitroso compounds.

\section{W. J. HICKINBOTTOM.} E. W. Lambert.

Department of Chemistry,

University, Birmingham.

${ }^{1}$ Karrer, Ber., 48, 1398 (1915).

2 Reilly and Hickinbottom, J. Chem. Soc., 118, 99 (1918).

3 Hickinbottom, Nature, 131, 762 (1933).

\section{Enzymatic Removal of Bound Carbohydrate from Normal Horse Serum Pseudoglobulin}

By the use of the preparation 'Luizyme' (Luitpoldwerke, Munich, to which I am indebted for a sample) part of the bound carbohydrate can be removed from horse serum pseudoglobulin. The enzyme is active at $p H 5$ in $M / 2$ acetate buffer. Owing to inhibition by the products of digestion, the action comes to an end within four hours at $37^{\circ}$, and it was necessary to submit the protein to successive treatment with fresh lots of enzyme. Ammonium sulphate (40 per cent saturated) was used at every stage as protein precipitant, and repeated reprecipitation was shown not to effect removal of carbohydrate. The slight proteolytic activity of the enzyme pre. paration under these conditions is quite insufficient to account for the splitting of carbohydrate from the protein.

The effect of successive digestions, shown in the accompanying table, on the amount of bound carbohydrate estimable by the method of Sørensen and Haugaard ${ }^{1}$, suggests a specific removal of one part of the galactose-mannose-glucosamine complex. The

No. of successive
digestions
1
5
5
8
10
Per cent of total estimable carbohydrate remaining ( \pm 3 per cent)

80
56
51
50
49

protein recovered after ten digestions did not differ markedly in its properties from the original protein, either in solubility, in ultra-violet absorption or in its potentiometric titration curve. Ultra-centrifugal sedimentation showed it to be rather heterogeneous, though at least a third of it had an unchanged sedimentation velocity. 